\title{
InGaAsP photonic crystal nanocavities with a Fano line shape resonant at $1.55 \mathrm{~m}$
}

\author{
Yu, Yi; Ek, Sara; Heuck, Mikkel; Yvind, Kresten; Mørk, Jesper
}

Published in:

Advanced Photonics Congress

Publication date:

2012

Document Version

Publisher's PDF, also known as Version of record

Link back to DTU Orbit

Citation (APA):

Yu, Y., Ek, S., Heuck, M., Yvind, K., \& Mørk, J. (2012). InGaAsP photonic crystal nanocavities with a Fano line shape resonant at $1.55 \mathrm{~m}$. In Advanced Photonics Congress (pp. IW3C.4). Optical Society of America.

\section{General rights}

Copyright and moral rights for the publications made accessible in the public portal are retained by the authors and/or other copyright owners and it is a condition of accessing publications that users recognise and abide by the legal requirements associated with these rights.

- Users may download and print one copy of any publication from the public portal for the purpose of private study or research.

- You may not further distribute the material or use it for any profit-making activity or commercial gain

- You may freely distribute the URL identifying the publication in the public portal

If you believe that this document breaches copyright please contact us providing details, and we will remove access to the work immediately and investigate your claim. 


\title{
InGaAsP photonic crystal nanocavities with a Fano line shape resonant at $1.55 \mu \mathrm{m}$
}

\author{
Yi Yu, Sara Ek, Mikkel Heuck, Kresten Yvind and Jesper Mørk \\ DTU Fotonik,, Technical University of Denmark, DK-2800 Kongens Lyngby, Denmark. \\ jesm@fotonik.dtu.dk
}

\begin{abstract}
We fabricated and characterized InGaAsP photonic crystal nanocavities. By carefully tailoring the structural parameters, both an efficient coupling and a suitable Q-factor can be achieved. Depending on the design of the coupling region, sharp Fano lines may be observed. OCIS codes: (050.5298) Photonic crystals; (350.4238) Nanophotonics and photonic crystals; (220.4241) Nanostructure fabrication.
\end{abstract}

\section{Introduction}

Photonic crystal (PhC) structures employing tailored dispersion and nanocavities are promising for tailoring lightmatter interactions and realizing signal processing facilities, such as ultra-fast and low-energy switching, in compact photonic integrated circuits. PhC nanocavity devices have already been fabricated using various materials [1]-[3]. In this paper we present results on InGaAsP PhC structures employing H0 cavities. By carefully adjusting the structural parameters, both an efficient coupling and a suitable Q-factor can be achieved. Furthermore, we find that depending on the design of the coupling region, Fano lines may appear, relying on the presence of additional reflections in the waveguide. Surprisingly strong undulations of the transmission spectra and very sharp Fano lines are observed.

\section{Device fabrication and characterization}

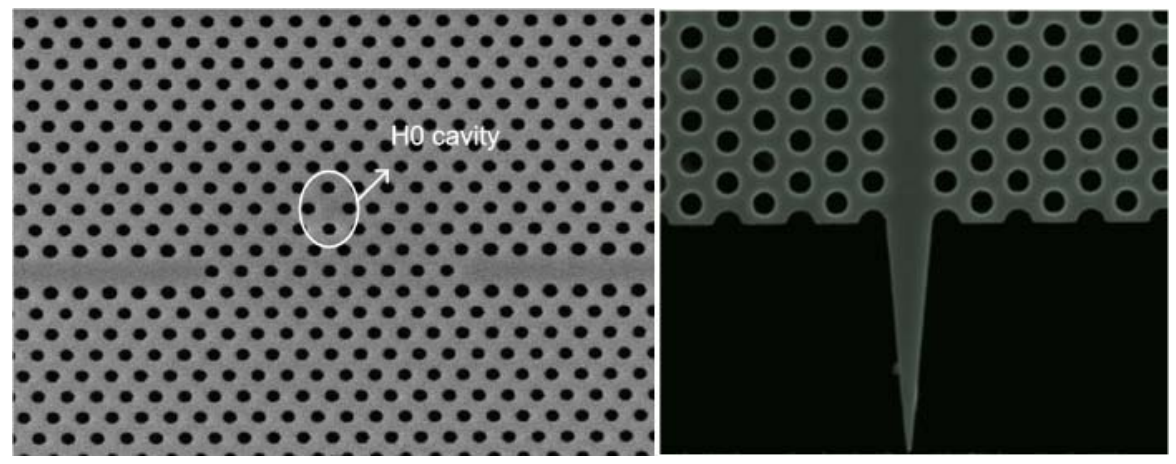

Fig. 1 SEM images of the fabricated photonic crystal nanocavity. (a) H0 cavity and (b) waveguide with taper.

The device is a membrane structure based on InGaAsP material which is commonly used for communication applications operating in the $1.33 / 1.55 \mu \mathrm{m}$ regime. The air holes are first patterned into the substrate by electronbeam lithography using an electron-beam writer (JEOL-JBX9300FS) on positive resist (ZEP520A), then transferred to a $200 \mathrm{~nm} \mathrm{Si} \mathrm{N}_{4}$ by $\mathrm{CHF}_{3} / \mathrm{O}_{2}$ reactive-ion etching (RIE), and further transferred to the semiconductor by a combination of $\mathrm{CH}_{4} / \mathrm{H}_{2}$ and $\mathrm{O}_{2}$ RIE. The membrane structure is finally formed with $\mathrm{HCl} / \mathrm{HF}$ selective wet-etching. Fig. 1 shows scanning electron microscope (SEM) images of the fabricated photonic crystal structure. The H0 cavity, which is formed by shifting two neighboring holes in opposite directions, is coupled with input and output $\mathrm{PhC}$ waveguides in the $\Gamma-\mathrm{K}$ direction. The size of the two arrays of the holes nearest to the waveguide is enlarged. Both in- and output- facets of the $900 \mu \mathrm{m}$ long PhC are equipped with tapers to reduce facet reflections [4]. Several samples with different structural parameters are fabricated. 


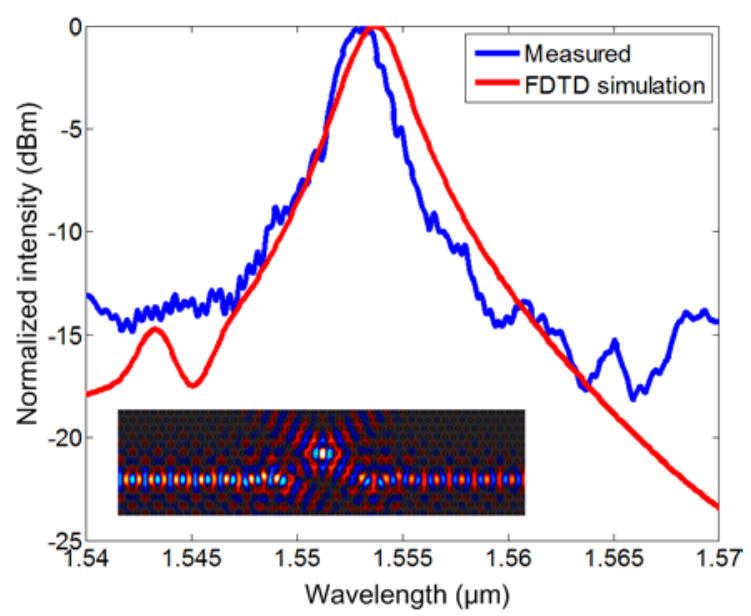

Fig. 2 Measured (blue line) and simulated (red line) transmission spectrum of the photonic crystal nanocavity. The inset shows the magnetic field profile of the device at $1.553 \mu \mathrm{m}$, as obtained from FDTD simulations.

The fabricated devices are characterized using a tunable CW laser source. The light is TE-polarized and is coupled into the device using a lensed single mode fiber, finally collected by another lensed single mode fiber at the output and sent to an optical spectrum analyzer (400-1750 nm, Ando AQ-6317B). The normalized transmittance of one of our devices is shown in Fig. 2. The hole diameter, the lattice constant and the slab thickness are 184, 390 and $340 \mathrm{~nm}$, respectively. The shift of the holes in H0 cavity is $78 \mathrm{~nm}$. The diameter of the holes nearest to the waveguide is $202 \mathrm{~nm}$. In Fig. 2, the red curve is the corresponding finite-difference time-domain (FDTD) simulation result. The magnetic field profile of the PhC nanocavity is also computed at $1.553 \mu \mathrm{m}$ and shown in the inset of Fig. 2. The device has an insertion loss of only $-11 \mathrm{~dB}$ including both coupling and propagation losses. The cavity Qfactor is 630 which means that this type of structure is suitable for signal processing at bitrates up to $300 \mathrm{~Gb} / \mathrm{s}$. From Fig. 2 we see that our calculation result agrees very well with the experimental measurements, and by carefully adjusting the structural parameters, both an efficient coupling and a suitable Q-factor can thus be achieved.
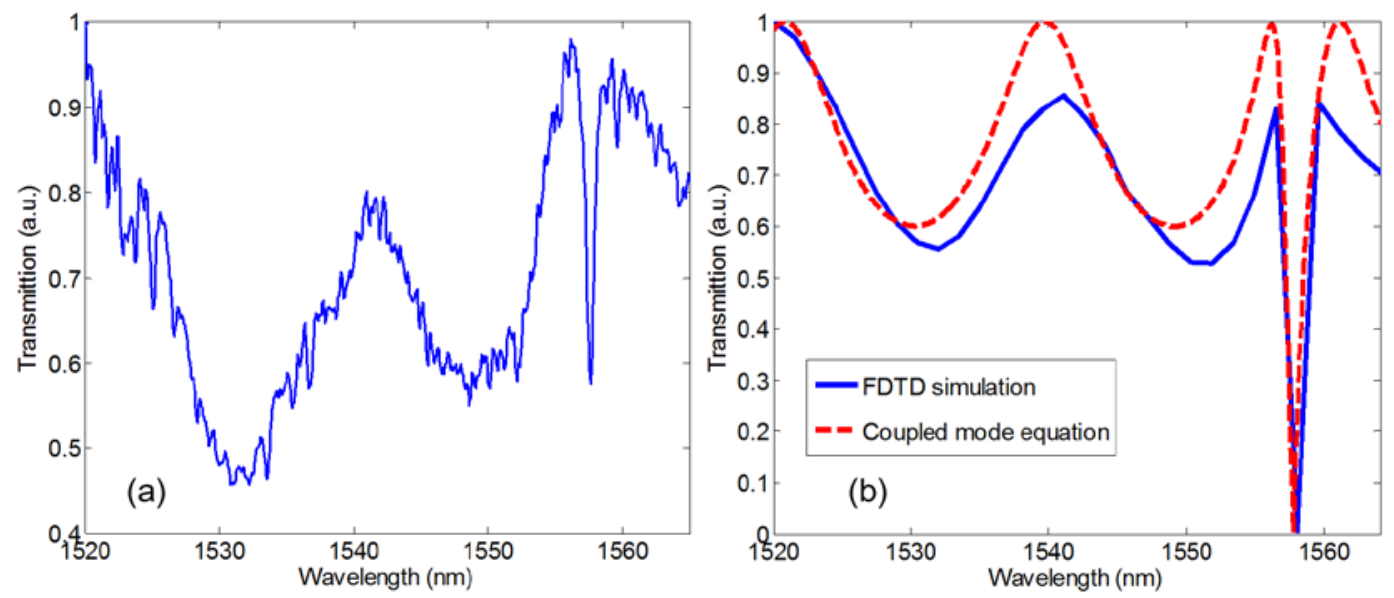

Fig. 3 (a) Measured, (b) FDTD and coupled mode theory calculated transmission spectrum of the photonic crystal nanocavity.

From the transmission measurements, we also find that some of the devices exhibit Fano line resonances, as shown in Fig. 3 (a). The device for which results are shown in Fig. 3 (a) has a hole diameter, lattice constant and cavity hole shift of 168, 380 and $88 \mathrm{~nm}$, respectively. The holes nearest to the waveguide have the diameter of 198 $\mathrm{nm}$. The radius of the 9 holes in the middle of the waveguide is reduced to $70 \mathrm{~nm}$, which makes most of the light directly transmit through the waveguide, and the device then acts as a side-coupled system rather than an in-line one. In order to find the origin of this Fano resonance and the nearly periodic variations of the transmission in the neighborhood of the resonance, both FDTD simulations and simulations based on coupled mode theory [5] are carried out. 


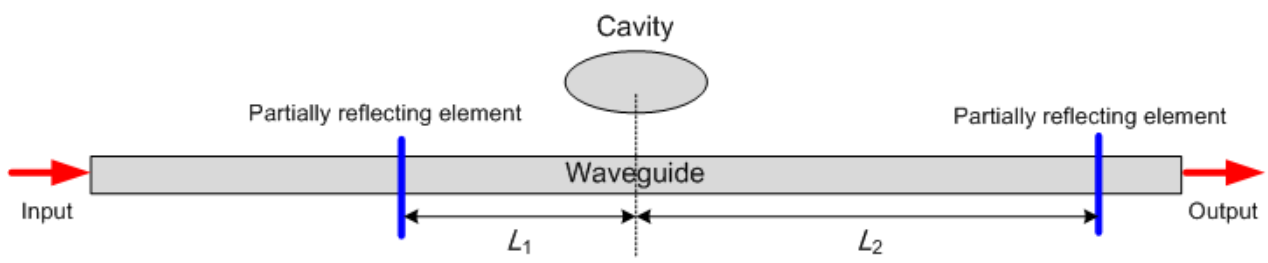

Fig. 4 Schematic of a side-coupled system including a waveguide coupled to a cavity. Two partially reflecting elements are placed in the waveguide.

For the side-coupled system shown in Fig. 4, the transfer matrix for the system $T$ can be described as

$$
T=-\frac{1}{1-r^{2}}\left[\begin{array}{cc}
-1 & -r \\
r & 1
\end{array}\right]\left[\begin{array}{cc}
e^{i b} & 0 \\
0 & e^{-i b}
\end{array}\right]\left[\begin{array}{cc}
1-\frac{i \gamma}{\omega-\omega_{0}} & \frac{-i \gamma}{\omega-\omega_{0}} \\
\frac{i \gamma}{\omega-\omega_{0}} & 1+\frac{i \gamma}{\omega-\omega_{0}}
\end{array}\right]\left[\begin{array}{cc}
e^{i a} & 0 \\
0 & e^{-i a}
\end{array}\right]\left[\begin{array}{cc}
-1 & -r \\
r & 1
\end{array}\right]
$$

where $r$ is the amplitude reflectivity of the partially reflecting element, $\omega_{0}$ and $\gamma$ are the center frequency and the width of the resonance of the cavity, $\omega$ is the frequency of input light. $a, b$ are the phase shifts from the partially reflecting element to the cavity, and $a=\omega n_{\text {eff }} L_{1} / c, b=\omega n_{\text {eff }} L_{2} / c$. From Eq. (1), the amplitude transmissivity $t$ is determined as

$$
t=\frac{\left(r^{2}-1\right) e^{i(a+b)}\left(\omega-\omega_{0}\right)}{-r^{2} e^{2 i(a+b)}\left(\omega-\omega_{0}-i \gamma\right)-r\left(e^{2 i a}+e^{2 i b}\right)(i \gamma)+\omega-\omega_{0}+i \gamma}
$$

Fig. 3 (b) shows the simulation results based on FDTD as well as the predictions by Eq. (2) from which we find that there should be a partially reflecting element in the waveguide. By setting $L_{1}=0$, the distance $L_{2}$ between the reflecting element near the output and the middle of the waveguide is about $15 \mu \mathrm{m}$. This reflecting element and the holes in the middle of the PhC waveguide form a Fabry-Perot like cavity, in which the continuum states interfere with the discrete energy state of the H0 cavity to give rise to a sharp Fano resonance. Through the coupled mode calculation, the field amplitude reflectivity of this Fabry-Perot cavity is predicted to be around 0.5, and the product of the Fabry-Perot cavity length and the effective index $L \times n_{\text {eff }}$ is about $62 \mu \mathrm{m}$. Currently, the origin of this partially reflecting element is not clear, but it may be caused by stitching errors incurred during electron-beam lithography. Such Fano resonances can significantly reduce the frequency shift needed in optical switches, and engineered structures may be exploited to lower the switching energy.

\section{Conclusion}

We fabricated and characterized InGaAsP photonic crystal nanocavities. By carefully tailoring the structural parameters, both an efficient coupling and a suitable Q-factor can be achieved. Furthermore, we find that depending on the design of the coupling region, Fano lines may appear, relying on the presence of an additional reflection in the waveguide, although the formation of this partially reflecting element is not presently clear. Further characterization results including the investigation of structures engineered to exploit the Fano resonance for lowering the switching energy will be reported.

\section{Acknowledgements}

The authors acknowledge financial support from Villum Fonden via the NATEC (NAnophotonics for TErabit Communications) Centre.

\section{References}

[1] P. E. Barclay, K. Srinivasan, and O. Painter, "Nonlinear response of silicon photonic crystal microresonators excited via an integrated waveguide and fiber taper,” Opt. Express 13, 801-820 (2005).

[2] C. Husko, A. De Rossi, S. Combrié1, Q. V. Tran, F. Raineri, and C. W. Wong, "Ultrafast all-optical modulation in GaAs photonic crystal cavities,” Appl. Phys. Lett. 94, 021111 (2009).

[3] K. Nozaki, T. Tanabe, A. Shinya, S. Matsuo, T. Sato, H. Taniyama and M. Notomi, "Sub-femtojoule all-optical switching using a photoniccrystal nanocavity,” Nature Photonics 4, 477-483 (2010).

[4] Q. V. Tran, S. Combrié, P. Colman, and A. De Rossi, "Photonic crystal membrane waveguides with low insertion losses,” Appl. Phys. Lett. 95, 061105 (2009).

[5] S. H. Fan, “Sharp asymmetric line shapes in side-coupled waveguide-cavity systems," Appl. Phys. Lett. 80, 908-910 (2002). 